\title{
Ultrafast deactivation processes in the 2-aminopyridine dimer and the adenine-thymine base pair: Similarities and differences
}

\author{
Yue-Jie Ai, ${ }^{1,2}$ Feng Zhang, ${ }^{1,2}$ Gang-Long Cui, ${ }^{1}$ Yi Luo, ${ }^{2,3, a)}$ and Wei-Hai Fang ${ }^{1, b)}$ \\ ${ }^{1}$ College of Chemistry, Beijing Normal University, Beijing 100875, China \\ ${ }^{2}$ Theoretical Chemistry, School of Biotechnology, Royal Institute of Technology, S-10691 Stockholm, Sweden \\ ${ }^{3}$ Department of Chemical Physics, Hefei National Laboratory for Physical Sciences at the Microscale, \\ University of Science and Technology of China, Hefei, Anhui 230026, China
}

(Received 1 June 2010; accepted 23 June 2010; published online 11 August 2010)

\begin{abstract}
2-aminopyridine dimer has frequently been used as a model system for studying photochemistry of DNA base pairs. We examine here the relevance of 2-aminopyridine dimer for a Watson-Crick adenine-thymine base pair by studying UV-light induced photodynamics along two main hydrogen bridges after the excitation to the localized ${ }^{1} \pi \pi^{*}$ excited-state. The respective two-dimensional potential-energy surfaces have been determined by time-dependent density functional theory with Coulomb-attenuated hybrid exchange-correlation functional (CAM-B3LYP). Different mechanistic aspects of the deactivation pathway have been analyzed and compared in detail for both systems, while the related reaction rates have also be obtained from Monte Carlo kinetic simulations. The limitations of the 2-aminopyridine dimer as a model system for the adenine-thymine base pair are discussed. (C) 2010 American Institute of Physics. [doi:10.1063/1.3464485]
\end{abstract}

\section{INTRODUCTION}

The photostability under the ultraviolet (UV) irradiation is one of the most remarkable properties of DNA, which has played an important evolutionary role in protecting the genetic information against photodamages and in avoiding serious problems in the transcription and other biological processes. However, solar ultraviolet light can still create excited electronic states in DNA that lead to mutagenic photoproducts. It has been shown that many DNA photolesions, including bipyrimidine photodimer, are formed from singlet excited-states. ${ }^{1-4}$ Understanding the structure and properties of the initial excited singlet electronic state is essential in elucidating the photostable mechanism in DNA. Because of extremely short lifetimes of the singlet excited-states and complex combinations of several tautomeric forms of the base pairs, experimental studies on real DNA base pair are very difficult.

Various model systems to mimic DNA base pairs have thus been suggested over the years. Among them, 2-aminopyridine (AP) dimer, see Scheme 1, is probably the most widely used model for investigating basic photochemical reaction mechanisms. ${ }^{5-9}$ This dimer consists of the $\mathrm{N}-\mathrm{H}$ donor and the aromatic $\mathrm{N}$ acceptor group of the single base and is also of doubly hydrogen-bonded structure. Earlier theoretical studies on one-dimensional potential-energy surfaces of excited-states in the 2-AP dimer ${ }^{7}$ have shown that a conical intersection between a local excited-state (LE) and a charge transfer (CT) state along a proton-transfer coordinate was responsible for the deactivation of the 2-AP dimer in vacuum. This electron-driven proton-transfer process was considered to be the reason behind experimentally observed

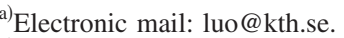

${ }^{b)}$ Electronic mail: fangwh@bnu.edu.cn.
}

short lifetime of the excited-state (65 \pm 10 picoseconds) of the 2-AP dimer. ${ }^{5}$ Later theoretical studies on onedimensional potentials of Watson-Crick adenine-thymine ${ }^{10}$ (A-T) and guanine-cytosine ${ }^{11}$ (G-C) base pairs revealed the same deactivation pathways via excited-state proton-transfer, which seem to suggest that the 2-AP dimer is a reasonable model for the real DNA base pair.

However, there are limitations in the earlier models. For instance, previous theoretical calculations only considered one $\mathrm{N}-\mathrm{H}$ bond as the reaction coordinate. In a doubly hydrogen bond system, a quantitative study of the hydrogen or proton transfer process needs the knowledge of at least a reliable two-dimensional potential-energy surface (2D PES) involving two hydrogen bond or proton coordinates. Moreover, in DNA oligomers, the base pairs are covalently bonded to the sugar-phosphate backbone which restricts the intermolecular distance of the base pairs. This structural constrain that cannot be enforced in a 2-AP dimer might have significant impacts on the electronic structure of the DNA base pair.

In the present work, we have examined the relevance of the 2-aminopyridine dimer as a model for the A-T base pair (Scheme 1) by comparing 2D-PESs of their excited-states obtained from the same computational methods, which allows to directly demonstrating the similarities and differences between these two systems. We focus on the ground state, low-lying local excited-state, and charge transfer states along the two hydrogen/proton transfer reaction coordinates. Time-dependent density functional theory (TDDFT) has been employed with Coulomb-attenuated hybrid exchangecorrelation functional (CAM-B3LYP). ${ }^{12}$ CAM-B3LYP is specially designed for treating the long-range $\mathrm{CT}$ transitions, and it has been successfully used in recent CT transition study of the A-T base pair. ${ }^{13}$ Based on the calculated 2D-PES 


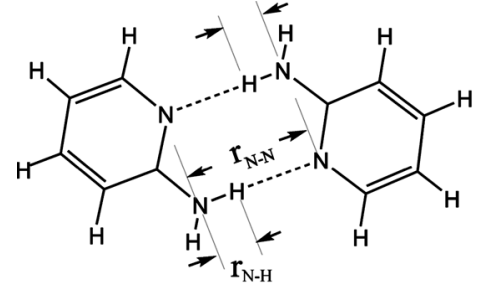

2-AP dimer

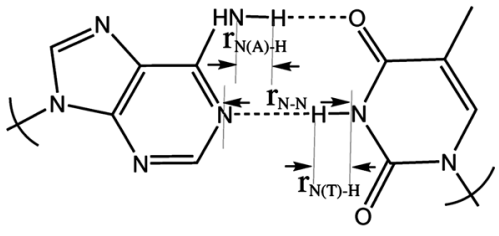

A-T base pair

SCHEME 1. 2-AP dimmer; A-T base pair.

of the two systems, we have also carried out Monte Carlo simulations to examine the kinetics of the electron-driven proton-transfer process involved.

\section{COMPUTATIONAL METHODS}

\section{A. Equilibrium structures and potential-energy surfaces}

Equilibrium structures of the 2-AP dimer and the A-T base pair in the ground $\left(S_{0}\right)$ state are optimized at B3LYP/ 6-31G $(\mathrm{d}, \mathrm{p})$ level without any geometrical constraints. For the construction of the 2D PES, the reaction path for proton or hydrogen-transfer has adopted the coordinate-driven minimum-energy path, i.e., for a given $\mathrm{NH}$ bond length or fixed $\mathrm{N} \cdots \mathrm{N}$ distance, all remaining coordinates were optimized. Based on the optimized geometries of the ground state, the potential-energy surfaces for the excited-states of interest along the reaction path have been calculated by TDDFT method with CAM-B3LYP functional. ${ }^{12}$ Since accurate high level calculations such as CASPT2 and CASSCF employing large active spaces are tedious and timeconsuming, TDDFT is gaining increasing popularity as an efficient tool to calculate electronic excitations in biochemistry systems because of their simplicity and moderate computational cost. For the DNA base pair, there were also some excellent TDDFT works before which confirmed the availability for the A-T base pair. ${ }^{14,15}$ It is noted that B3LYP functional has also been used to calculate the excitation energies and the results, in particular, for CT states, are not particularly satisfied. In this work, TDDFT calculations with CAMB3LYP have been performed with DALTON $2.0^{16}$ program, while geometry optimizations of the ground states are done with GAUSSIAN 03 software packages. ${ }^{17}$

\section{B. Microcanonical Monte Carlo rate constant}

The microcanonical rate constant $\mathrm{k}$ is given as

$$
k=\frac{1}{2} \frac{N(E)}{h \rho(E)},
$$

where $\rho(E)$ is the density of reactant states per unit energy and $N(E)$ is the number of states. The factor $(1 / 2)$ comes from the fact that only one-way crossing can contribute to the rate constant. In the microcanonical ensemble, the density of states is given by

$$
\rho(E)=\frac{1}{h^{3 N} N !} \int \delta\left[E-H\left(p^{N}, q^{N}\right)\right] d p^{N} d q^{N} .
$$

The integral over momentum can be solved analytically by separating the variables, when the density is written in the configuration space,

$$
\begin{aligned}
\rho(E)= & \frac{1}{\Gamma\left(\frac{3 N}{2}\right) N !}\left(\frac{\sqrt{2 \pi m}}{h}\right)^{3 N} \int\left[E-V\left(q^{N}\right)\right]^{(3 N-2) / 2} h \\
& \times\left[E-V\left(q^{N}\right)\right] d q^{N} .
\end{aligned}
$$

So the configuration probability distribution for the microcanonical ensemble is given by

$$
f(E) \propto\left[E-V\left(q^{N}\right)\right]^{(3 N-2) / 2} h\left[E-V\left(q^{N}\right)\right] .
$$

Then the rate constant can be written in the form of summation of separated-state numbers by using the Monte Carlo method. In our studies, a nonadiabatic transition from the reactant (LE state) to the product (CT state) may occur at a configuration lying in the region of the crossing seam. A schematic PES model for our Monte Carlo simulation is given in Scheme 2. The sampled configurations are generated on the LE state, while the PES of the CT state is only used for determining the crossing region. It looks like there is a small window in the LE state, the configurations located inside the window (the blue solid line) can jump to the product region (CT state) with $100 \%$ probability.

Finally, the nonadiabatic rate can be obtained by counting states lying in the crossing seam $\delta(S)$, where $S$ is defined as the potential-energy difference of the reactant and product, $S=V_{1}-V_{2}$,

$$
k(E)=\frac{1}{2} \frac{\sum_{i}^{M} h\left[E-V_{1}(i)\right] \delta\left(S_{i}\right)}{h \rho(E)} .
$$

The density of states $\rho(E)$ is the derivative of state numbers at the total energy $E$, which could be obtained by numerical

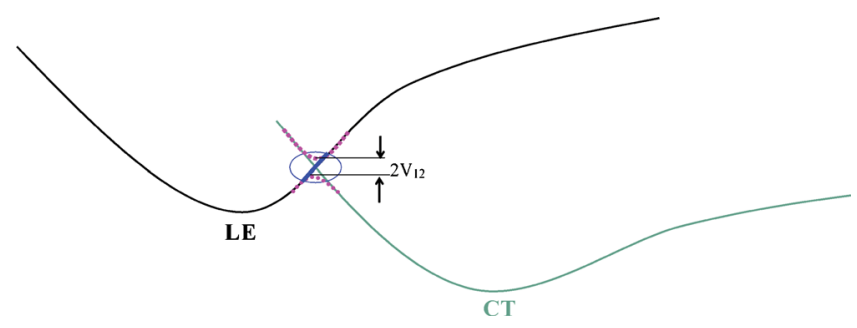

SCHEME 2. Schematic draw for Monte Carlo simulations. 

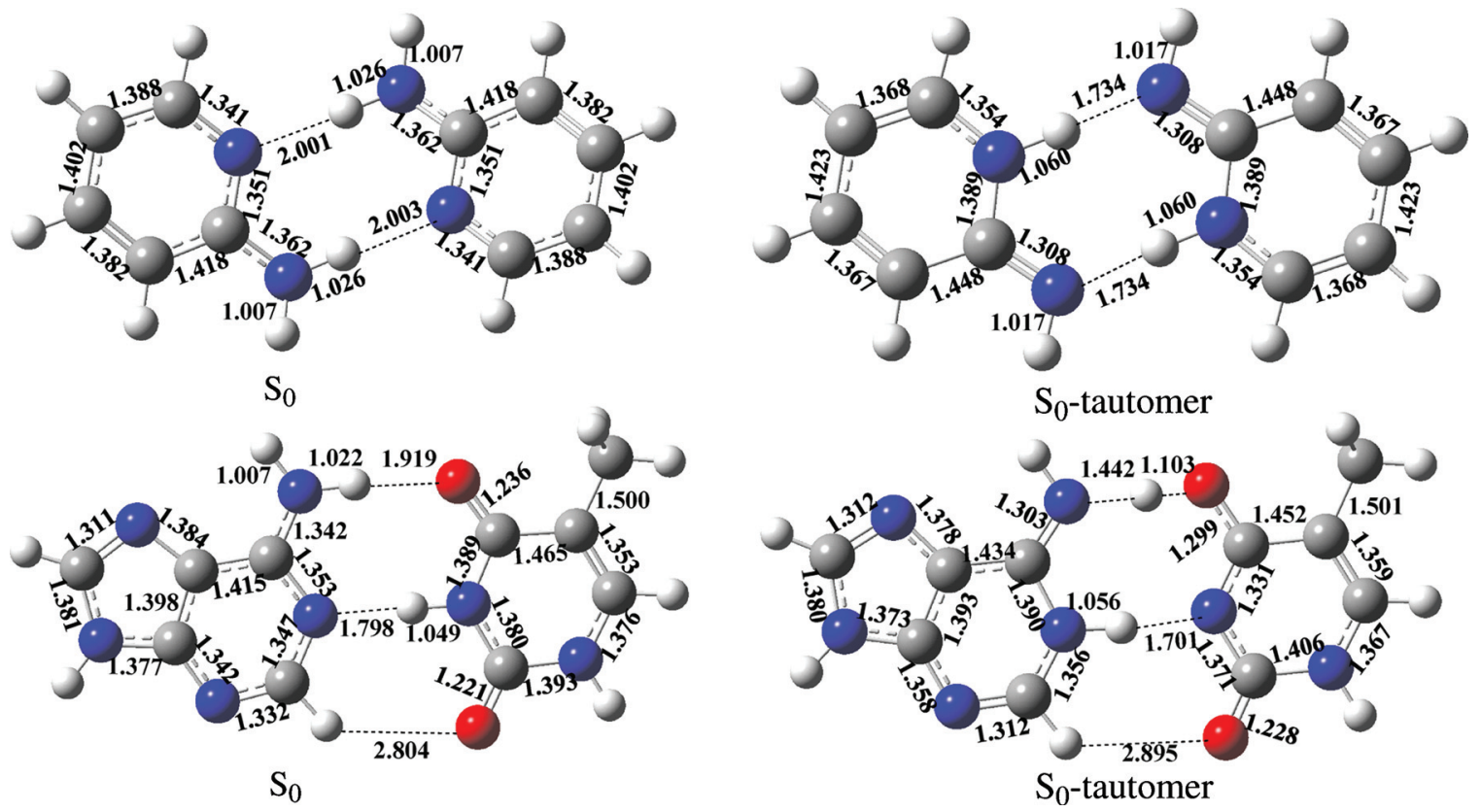

FIG. 1. Structures of stationary points for the 2-AP dimer and the A-T base pair in the ground state $\left(S_{0}\right)$. Some selected bond parameters are given.

calculations. Practically the Monte Carlo summation in Eq. (5) is obtained by using the important sampling technique. ${ }^{18,19}$

\section{RESULTS AND DISCUSSION}

\section{A. Ground state geometry}

The optimized structures for the 2-AP dimer and the A-T base pair in the $S_{0}$ state are depicted in Fig. 1. It can be seen that the 2-AP dimer is formed through intermolecular hydrogen-bond bridges. The $\mathrm{r}_{\mathrm{N}-\mathrm{H}}$ in the hydrogen bond bridge of the 2-AP dimer is $1.026 \AA$, which is $0.019 \AA$ longer than other nonhydrogen bonded $\mathrm{N}-\mathrm{H}$ bond. For the two main hydrogen bridges in the A-T base pair: $\mathrm{N}(\mathrm{A})-\mathrm{H} \cdots \mathrm{O}(\mathrm{T})$ and $\mathrm{N}(\mathrm{T})-\mathrm{H} \cdots \mathrm{N}(\mathrm{A})$, the lengths of the connected $\mathrm{N}-\mathrm{H}$ bond are 1.022 and $1.049 \AA$, respectively in the $S_{0}$ state. The $\mathrm{r}_{\mathrm{N}-\mathrm{N}}$ distance between adenine and thymine molecules is $2.846 \AA$, which is shorter than that in the 2-AP dimer (3.030 ̊). The two hydrogen-transfer products of these two molecules in the ground state are represented as their "tautomers" shown in Fig. 1. The $\mathrm{r}_{\mathrm{N}-\mathrm{H}}$ in the tautomer of the 2-AP dimer is 1.734 $\AA$, while the corresponding $\mathrm{N}-\mathrm{H}$ bonds in hydrogen-transfer tautomer of the A-T are 1.442 and $1.701 \AA$, respectively.

\section{B. Potential-energy surface of the ground state}

For the 2-AP dimer, based on the stationary structure in the ground state, we have optimized 225 geometries with a frozen $\mathrm{NH}$ distance $\left(\mathrm{r}_{\mathrm{N}-\mathrm{H}}\right)$ between two adjacent 2-aminopyridine molecules, which takes value from 0.8 to $2.2 \AA$ with a step of $0.1 \AA$. In other words, there are $15^{*} 15$ grid points for constructing 2D PES of the $S_{0}$ state. The numerical method of $2 \mathrm{D}$ cubic spline interpolation has then been applied to obtain smooth potentials, see Fig. 2(a). The two reaction coordinates are related to hydrogen positions in the two $\mathrm{N}-\mathrm{H}$ hydrogen bridges of the 2-AP dimer. There are two obvious minima in the ground state PES, corresponding to the 2-AP dimer and its tautomer. The tautomer is 17.2 $\mathrm{kcal} / \mathrm{mol}$ higher in energy than the 2-AP dimer. The 2D PES of the ground state of the 2-AP dimer has a symmetrical characteristic along the diagonal line due to its symmetric geometry.

Different from the 2-AP dimer, in DNA oligomers, the base pair is bonded to the sugar-phosphate backbone via covalent bonding with a defined intermolecular distance. Recently, Samoylova et al. ${ }^{20}$ reported that the electron/protontransfer barrier is sensitive to the $\mathrm{N} \cdots \mathrm{N}$ distance in the 2-AP dimer. Considering this external restriction, we have studied the A-T base pair in two different ways, that is, with free and with fixed intermolecular $\mathrm{N} \cdots \mathrm{N}$ distance following the coordinate-driven minimum-energy path as mentioned above. In this case, for a given value of $\mathrm{N}(\mathrm{A})-\mathrm{H} \cdots \mathrm{O}(\mathrm{T})$ $\left(\mathrm{r}_{\mathrm{N}(\mathrm{A})-\mathrm{H}}\right)$ or $\mathrm{N}(\mathrm{T})-\mathrm{H} \cdots \mathrm{N}(\mathrm{A})\left(\mathrm{r}_{\mathrm{N}(\mathrm{T})-\mathrm{H}}\right)$, or $\mathrm{N} \cdots \mathrm{N}$ distance all remaining coordinates were optimized.

The calculated 2D PES for the case of free $\mathrm{N} \cdots \mathrm{N}$ distance is given in Fig. 2(b). There are two minima with a very flat central transition region. The hydrogen-transfer path is not along the diagonal line. The corresponding $\mathrm{N}-\mathrm{H}$ bonds in the hydrogen-transfer tautomer are 1.442 and $1.701 \AA$, respectively. The energy of the tautomer is found to be 13.2 $\mathrm{kcal} / \mathrm{mol}$ higher than that of the initial A-T base pair. We have also found a transition state for the ground state hydrogen-transfer process. The hydrogen bonds, $\mathrm{r}_{\mathrm{N}(\mathrm{A})-\mathrm{H}}$ and $\mathrm{r}_{\mathrm{N}(\mathrm{T})-\mathrm{H}}$, of the transition state along the $\mathrm{N}(\mathrm{A})-\mathrm{H} \cdots \mathrm{O}(\mathrm{T})$ and $\mathrm{N}(\mathrm{T})-\mathrm{H} \cdots \mathrm{N}(\mathrm{A})$ hydrogen bridges are 1.42 and $1.69 \AA$, respectively. The transition state in the $S_{0}$ state has almost the same energy as its tautomer.

Figure 2(c) illustrates the projected two-dimensional ground state PES of the A-T base pair with a fixed intermolecular distance $\mathrm{r}_{\mathrm{N}(\mathrm{A}) \ldots \mathrm{N}(\mathrm{T})}\left(\mathrm{r}_{\mathrm{N}-\mathrm{N}}\right)=3.0 \AA$. In this case, the PES of the ground state is much steep. It is also possible to locate the transition state between these two minima at the coordinate near $\mathrm{r}_{\mathrm{N}(\mathrm{A})-\mathrm{H}}=1.1 \AA, \mathrm{r}_{\mathrm{N}(\mathrm{T})-\mathrm{H}}=1.6 \AA$. The barrier 


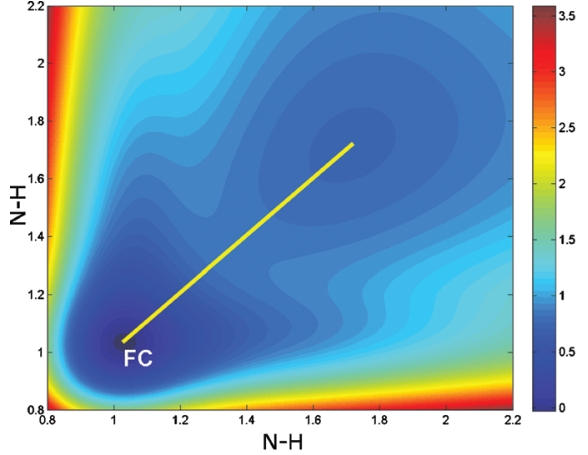

(a)

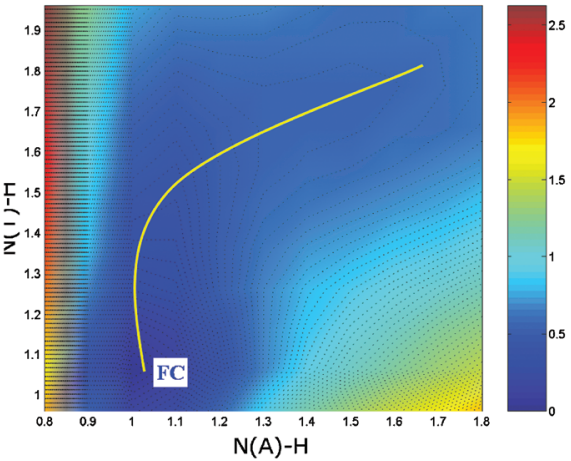

(b)

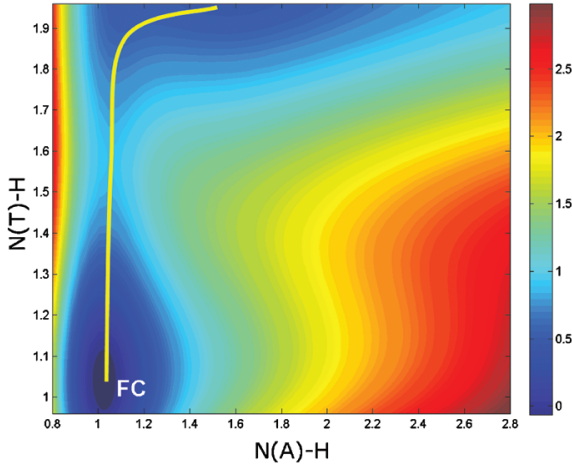

(c)

FIG. 2. Two-dimensional potential-energy surfaces of the ground state for (a) 2-AP dimer and for the A-T base pair (b) with free and (c) fixed N... N distance. The picture is obtained from MATLAB 7.1.

of this hydrogen-transfer pathway is $23.5 \mathrm{kcal} / \mathrm{mol}$, which is almost $10 \mathrm{kcal} / \mathrm{mol}$ higher than those with a free $\mathrm{N} \cdots \mathrm{N}$ distance. One can at least conclude that for the A-T base pair, the reaction barrier in the ground state is very sensitive to the intermolecular $\mathrm{N} \cdots \mathrm{N}$ bond distance.

For both 2-AP dimer and A-T base pair, many studies have been devoted to the hydrogen- and proton-transfer mechanisms in the ground and excited-states. ${ }^{15}$ However, no consensus has been reached on whether it should involve a single or double hydrogen (or proton) transfer mechanism. As can be seen in Fig. 2(a), the hydrogen-transfer exhibits concerted mechanism in the ground state due to the symmetrical structure of the 2-AP dimer. Whereas from Figs. 2(b) and 2(c), we can conclude that the ground state hydrogen-transfer in the A-T base pair is a stepwise process. In this case, the hydrogen of $\mathrm{N}(\mathrm{T})-\mathrm{H}$ moves first with the other hydrogen bond $\mathrm{N}(\mathrm{A})-\mathrm{H}$ almost unchanged. Then the hydrogen of $\mathrm{N}(\mathrm{A})-\mathrm{H}$ moves and reaches the equilibrium position of the tautomer structure. These two-step mechanism agrees with the results of Villani. ${ }^{21}$ It is thus important to note that in terms of ground state dynamics, the 2-AP dimer and the A-T base pair have very different hydrogen-transfer mechanisms. One could conclude that the 2-AP dimer is at least not a good model for studying the ground state dynamics of the A-T base pair.

\section{Potential-energy surfaces of excited-states}

Based on the optimized ground state geometries of the 2-AP dimer and the A-T base pair, by scanning the coordinates along the two main hydrogen bonds, we have obtained two-dimensional potential-energy surfaces of excited-states from TDDFT calculations. It is worth to note that to the best of our knowledge, these are the first complete twodimensional PES of excited-states for these essential biological systems. Earlier studies provided mostly some particular minima and barrier points or one dimensional PES. 2D-PESs of the important singlet electronic excitations of the 2-AP dimer are shown in Fig. 3(a). In addition to the ground state $S_{0}$, a local ${ }^{1} \pi \pi^{*}$ excited-state on the monomer is marked as LE state in Fig. 3(a). Sobolewski et al. ${ }^{7}$ predicted a low-lying ${ }^{1} \pi \pi^{*}$ CT state, where one electron from the $\pi$ orbital of one molecule is promoted to the $\pi^{*}$ orbital of the other molecule. This state is labeled as CT1 state in Fig. 3(a). Symmetrically, the opposite electron transfer ${ }^{1} \pi \pi^{*}$ CT state of the 2-AP dimer is set as $\mathrm{CT} 1{ }^{\prime}$. Recently, Samolylova et al. ${ }^{20}$ identified the next higher ${ }^{1} \pi \pi^{*} \mathrm{CT}^{*}$ state which was about $1 \mathrm{eV}$ higher than the low-lying CT state by CASPT2. It was found that this second CT state may also attribute to the ultrafast electronic relaxation with $\tau \leq 50$ fs of the 2-AP dimer.

From our TDDFT calculations, we have also reproduced this CT state (labeled as CT2) which is also about $1 \mathrm{eV}$

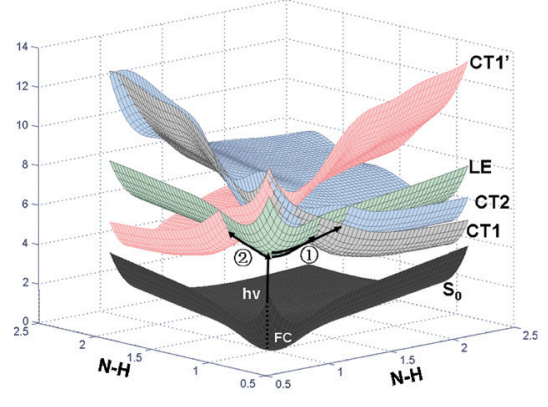

(a)

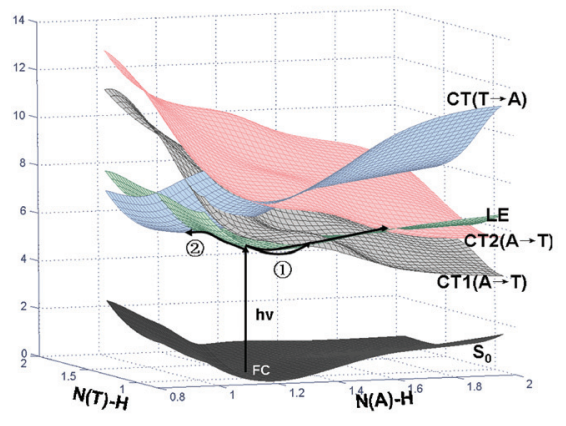

(b)

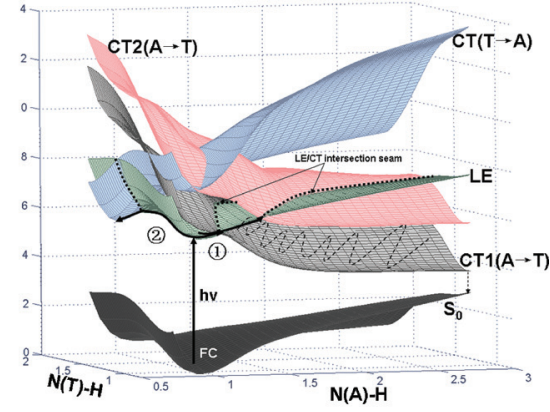

(c)

FIG. 3. Potential-energy surfaces of the ground $\left(S_{0}\right)$, CT, and localized (LE) states of (a) the 2-AP dimer; and the A-T base pair with (b) free and (c) fixed $\mathrm{N} \ldots \mathrm{N}$ distances. Two coordinates in the plan correspond to the main two hydrogen bridges, where the vertical coordinate is the energy in eV. 
TABLE I. Vertical excitation energy $(\Delta E)$ and oscillator strength (f) of some important excited singlet states of the 2-AP dimer and the A-T base pair involved in the electron-driven proton-transfer process. TDDFT at the CAM-B3LYP/6-31G(d,p) level is employed.

\begin{tabular}{lcccccc}
\hline \hline & \multicolumn{2}{c}{2 2-AP dimer } & & \multicolumn{3}{c}{ A-T base pair } \\
\cline { 2 - 3 } \cline { 5 - 6 } State & $\begin{array}{c}\Delta E \\
(\mathrm{eV})\end{array}$ & $\mathrm{f}$ & & & $\begin{array}{c}\Delta E \\
(\mathrm{eV})\end{array}$ & $\mathrm{F}$ \\
\hline LE & 4.81 & 0.14 & & LE & 5.48 & 0.11 \\
CT1 & 6.21 & 0.00 & & CT1 & 5.85 & 0.01 \\
CT2 & 7.00 & 0.00 & & CT2 & 7.35 & 0.00 \\
CT1 & 6.21 & 0.00 & & CT & 7.52 & 0.00 \\
\hline \hline
\end{tabular}

higher than the CT1 as shown in Fig. 3(a). The good agreement between our CAM-B3LYP and previous CASPT2 results ${ }^{20}$ highlights the usefulness of this functional for such systems. It can be seen that the energy of the ${ }^{1} \pi \pi^{*}$ (LE) character increases along the $\mathrm{N}-\mathrm{H}$ reaction coordinate, while the CT states are essentially repulsive. There are crossing seams between the potential-energy of CT1 and CT2 states and the LE state at $\mathrm{N}-\mathrm{H}$ distances longer than 1.2 and $1.5 \AA$, respectively.

Similarly, we have performed TDDFT calculations for potential-energy surfaces of excited-states of the A-T base pair. The results for the A-T base pair with free and frozen $\mathrm{N} \cdots \mathrm{N}$ distance are presented in Figs. 3(b) and 3(c), respectively. It is known from previous CIS and CC2/cc-pVDZ calculations that the character of the lowest ${ }^{1} \pi \pi^{*}$ (LE) state is of $\mathrm{A} \rightarrow \mathrm{A}^{*}$ type. ${ }^{10}$ However, from our TDDFT calculations with CAM-B3LYP, we have found two nearly degenerated LE states at 5.40 and $5.48 \mathrm{eV}$, of $\mathrm{T} \rightarrow \mathrm{T}^{*}$ type and $\mathrm{A} \rightarrow \mathrm{A}^{*}$ type, respectively. These two LE states are found to be always close in energy with very similar potential shapes. To simplify the discussion, we only present the LE state of A $\rightarrow \mathrm{A}^{*}$ character in Figs. 3(b) and 3(c). We have also given the results of two important charge transfer states, CT1 $(\mathrm{A} \rightarrow \mathrm{T})$ and $\mathrm{CT} 2(\mathrm{~A} \rightarrow \mathrm{T})$, which are both ${ }^{1} \pi \pi^{*}$ charge transfer from the base $\mathrm{A}$ to the base $\mathrm{T}$, as well as the opposite charge transfer state from base $\mathrm{T}$ to base $\mathrm{A}$, marked as $\mathrm{CT}(\mathrm{T} \rightarrow \mathrm{A})$. The energies of the $\mathrm{LE}$ and $\mathrm{CT}(\mathrm{T} \rightarrow \mathrm{A})$ states increase along the $\mathrm{N}(\mathrm{A})-\mathrm{H}$ reaction coordinate. The strongly repulsive potentials of $\mathrm{CT} 1(\mathrm{~A} \rightarrow \mathrm{T})$ and $\mathrm{CT} 2(\mathrm{~A} \rightarrow \mathrm{T})$ states cross over with the LE state after $\mathrm{N}(\mathrm{A})-\mathrm{H}$ distance beyond 1.1 and $1.4 \AA$, respectively. Moreover, the $\mathrm{CT}(\mathrm{T} \rightarrow \mathrm{A})$ state crosses over the LE state when $\mathrm{N}(\mathrm{T})-\mathrm{H}$ distance is longer than $1.6 \AA$.

The PESs of the A-T base pair with a fixed $\mathrm{N} \cdots \mathrm{N}$ distance differ from those with a free $\mathrm{N} \cdots \mathrm{N}$ distance, as clearly demonstrated in Fig. 3(c). Basically their potential surfaces of the ground and excited states are steeper with higher reaction barriers. The constraint from the DNA backbone seems to have significant effects on the potential-energy surfaces, hence the related ultrafast deactivation processes. It is noted that such a structural constrain cannot be enforced in the 2-AP dimer.

The difference in potential-energy surfaces of excited states of the 2-AP dimer and the A-T base pair can be clearly distinguished from Figs. 3(b) and 3(c). To highlight the difference, we have listed the vertical excitation energies of several relevant excited states in both 2-AP dimer and the A-T base pair in Table I. It can be seen that the vertical excitation energy of the LE state in the 2-AP dimer is 4.81 $\mathrm{eV}$, which is about $0.6 \mathrm{eV}$ lower than that in the A-T base pair. The CT1 and its symmetric charge transfer state CT1' have the same high energy $(6.21 \mathrm{eV})$ due to the symmetry of the 2-AP dimer. The vertical energy $(7.00 \mathrm{eV})$ of the second charge transfer state CT2 is about $0.8 \mathrm{eV}$ higher in energy that CT1. In comparison with the 2-AP dimer, the CT1 (A $\rightarrow \mathrm{T})$ and $\mathrm{CT}(\mathrm{T} \rightarrow \mathrm{A})$ of the A-T base pair are different in vertical energy by as much as $1.7 \mathrm{eV}$. This energy difference can induce different reaction barriers which will be discussed later.

\section{Deactivation mechanism in the 2-AP dimer and the A-T base pair}

The given potential-energy surfaces for both 2-AP dimer and the A-T base pair provide a good opportunity to discuss and to compare deactivation mechanism involved in these systems. It is seen that upon the UV excitation at $274 \mathrm{~nm}$, the 2-AP dimer is mainly excited to the lowest singlet local excited state ${ }^{1} \pi \pi^{*}$ (LE). Based on the PES in Fig. 3(a), it can be anticipated that the excited electron on the LE state can overcome various barriers and reach the crossing seams between the CT1 (or the CT2) and the LE states. It can be further relaxed to the ground state through the fast internal conversion (IC) process at large $\mathrm{N}-\mathrm{H}$ distance. We set this pathway as the path 1 . Meanwhile, the crossing between the charge transfer state $\mathrm{CT}^{\prime}{ }^{\prime}$ of opposite direction and the LE state can be another efficient deactivation pathway-named as the path 2. Both paths are quite competitive because of the involvement of same barriers caused by symmetric characteristics of the CT1 and the CT1' states. With even shorter pump wavelengths, the electron can reach the surface of the CT2 that can directly relax along the $\mathrm{N}-\mathrm{H}$ stretch pathway.

With excitation wavelengths of $250-272 \mathrm{~nm}$, the A-T base pair is excited to the ${ }^{1} \pi \pi^{*}$ excited states which lies approximately $5 \mathrm{eV}$ above the ground state. ${ }^{22}$ After excitation to the LE states, there are also two possible relaxation paths involved. In contrast to the case of the 2-AP dimer, the notable difference in the relaxation process in the A-T base pair is that the barrier on the path 2 is much higher than that on the path 1 . As concluded by Domcke et al., ${ }^{10}$ the CT state which is of the $\mathrm{A} \rightarrow \mathrm{T}^{*}$ type corresponds to the formation of the $\mathrm{A}^{+}-\mathrm{T}^{-}$ion pair structure, which can drive the proton 


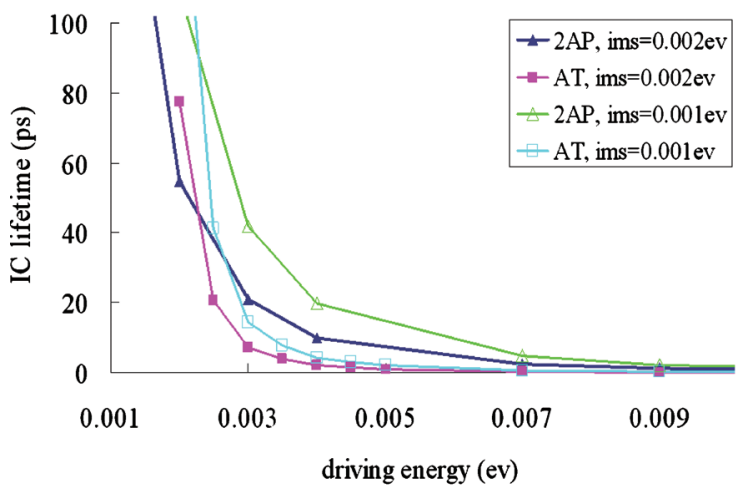

(a)

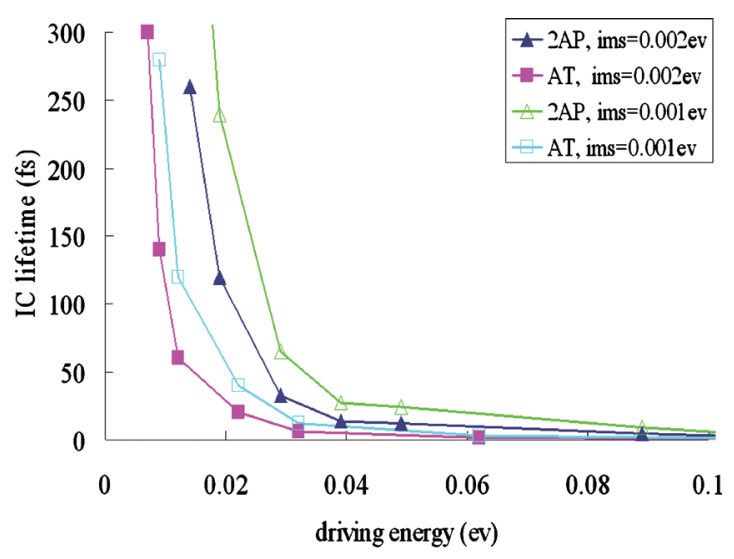

(b)

FIG. 4. Energy dependence of the LE1 $\rightarrow$ CT1 IC lifetime under the excitation of the (a) low $(<0.01 \mathrm{eV})$ and (b) high $(>0.01 \mathrm{eV})$ driving energies.

transfer from the base $\mathrm{A}$ to the base $\mathrm{T}$ to compensate the charge separation. However, the ionization potential of the base $\mathrm{T}$ is higher than that of the base A. This makes the deactivation path 2 less efficient than the path 1 . It means that the $\mathrm{CT}$ state of $\mathrm{A} \rightarrow \mathrm{T}^{*}$ type is energetically more favorable than the opposite $\mathrm{T} \rightarrow \mathrm{A}^{*}$ type of $\mathrm{CT}$ state. The latter state has a higher electron/proton transfer barrier as shown in Fig. 3(c). It is worth to notice that if the excitation energy is higher enough to reach the PES of the $\mathrm{CT}(\mathrm{T} \rightarrow \mathrm{A})$ state, the $\mathrm{CT}$ state of $\mathrm{T} \rightarrow \mathrm{A}^{*}$ type can then become a possible deactivation channel along the $\mathrm{N}(\mathrm{T})-\mathrm{H}$ coordinate.

It is clear that the electron-driven proton charge transfer state always plays an important role in the relaxation processes in the singlet excited ${ }^{1} \pi \pi^{*}$ (LE). The height of the barrier which arises as a consequence of the LE/CT crossing along the proton-transfer coordinate is a decisive factor in this rate-determining step in the ultrafast deactivation process.

\section{E. LE $\rightarrow C T$ internal conversion rate}

The PESs of electronic states in the 2-AP dimer and the A-T base pair are quite different in terms of energy position, energy separation, and potential shapes. It would be interesting to see how these differences affect the actual decay rates. For this purpose, we have carried out microcanonical Monte Carlo simulations to calculate the rate constant of the IC process via the intersection seam between the local excited (LE) state and the charge transfer (CT1) state. The transition from the LE to the CT1 state is obvious a nonadiabatic process in nature. The Landau-Zener nonadiabatic probability is often regarded as unit for an internal conversion process via the conical intersection. ${ }^{23}$ As indicated by Scheme 2, the size of the crossing region, as the ellipse zone marked in Scheme 2 , will influence the number of selected configurations located in the crossing seam region. In other words, the nonadiabatic coupling matrix element $\mathrm{V}_{12}$ is an important parameter for the microcanonical Monte Carlo rate constant. Unfortunately, the calculations of the nonadiabatic coupling matrix element for 2-AP dimer and AT molecule are far too complicated and beyond the capacity of the used programs.

Although accurate coupling matrix elements are not available, we can still discuss the effect of the coupling by a set of parameters, i.e., to highlight the relative changes. Our calculations indicate that the IC lifetime from the LE to the CT1 states is directly proportional to the nonadiabatic coupling between the two states. The IC lifetime of the 2-AP dimer and the A-T base pair as a function of driving energy are shown in Fig. 4, when $2 \mathrm{~V}_{12}$ (labeled as ims in Fig. 4) is chosen as 0.002 and $0.001 \mathrm{eV}$, respectively. The driving energy is defined as the excess energy of the system after overcoming the barrier of the minimum-energy crossing point. It is noted that the present Monte Carlo simulation is based on the classical concept without considering the quantum tunneling effect, which implies that the IC lifetime is infinity when the driving energy is less than zero. During the total $5 \times 10^{6}$ random moves, the minimum crossing point is located at $(1.35 \AA, 1.07 \AA, 5.012 \mathrm{eV})$ for the 2-AP dimer, and $(1.14 \AA, 1.00 \AA, 5.588 \mathrm{eV})$ for the A-T molecule. From Fig. 4, we can see that the IC lifetime is very sensitive to the driving energy. For clarity, the IC lifetime is presented into two regimes, at the low driving energy [Fig. 4(a)] and the high driving energy [Fig. 4(b)]. For both systems, the LE $\rightarrow$ CT1 IC lifetime decays very rapidly with the increase of the energy, as soon as the energy is enough to overcome the "barrier" of the crossing seam.

For the 2-AP dimer, the nonadiabatic transition from the LE to CT1 states is expected to occur in the time scale of dozens of picoseconds even when the total energy is only $0.001 \mathrm{eV}$ higher than the barrier. Moreover, the IC lifetime will be shortened to the scale of dozens of femtoseconds in case the driving energy is higher than $0.01 \mathrm{eV}$. The lifetime of the excited state of the 2-AP dimer has been measured to be $65 \pm 10$ ps by the femtosecond time-resolved mass spectroscopy experiment with $274 \mathrm{~nm}$ excitation. ${ }^{5,20}$ Our simulations might lead to two possible mechanistic interpretations for this experimental result: (a) the driving energy is sufficiently small in the experiment, which can result in long decay time in the scale of picoseconds; (b) there might be another conical intersection due to the slow structural relaxation. Further study is thus needed to resolve this puzzle. Obviously, the limitations of the Monte Carlo simulations used in this study could also be the possible source for the difference between the calculated and experimental lifetimes. 
Comparing with the 2-AP dimer, the IC process between the LE and the CT1 states of the A-T base pair displays a very similar trend: long lifetime (scale of picoseconds) with very low excitation energy and ultrafast decay (scale of femtoseconds) with the driving energy more than $0.01 \mathrm{eV}$. Actually, due to the lower barrier, the LE state of the A-T base pair decays more rapidly to the CT1 state than in the case of the 2-AP dimer with the driving energy. It can also be found that the difference in the shape of the PES can also lead to different decay rates in two systems.

\section{CONCLUSION}

In summary, we have provided a visual and more complete picture on the relevance of the 2-AP dimer as a model to mimic A-T base pair. Our calculations have shown that the 2-AP dimer model has limited value for understanding photochemistry of real A-T base pair since it lacks some important structural information about the A-T base pair. For instance, the hydrogen-transfer mechanism in the ground state of the 2-AP dimer is completely different from the A-T base pair. Moreover, the sugar-phosphate backbone in DNA double strands forces the base pair to be at a reasonable intermolecular distance while this structural constrain cannot be enforced in the 2-AP dimer. The fixed intermolecular distance can actually lower the reaction barrier and modifies the potential-energy surfaces. However, the specific difference in the PESs of these two systems seems to have very little effects on the kinetics of the IC process as demonstrated by Monte Carlo kinetic studies. For this particular purpose, the 2-AP dimer could be a reasonable model for the A-T base pair.

\section{ACKNOWLEDGMENTS}

The work was supported by the Swedish National Infrastructure for Computing (SNIC), the NSFC (Grant Nos.
20720102038 and 20925311) and the Major State Basic Research Development Programs (Grant Nos. 2004CB719903 and 2010CB923300) of China.

${ }^{1}$ J. Cadet and P. Vigny, The Photochemistry of Nucleic Acids in Bioorganic Photochemistry (Wiley, New York, 1990).

${ }^{2}$ A. A. Lamola and J. Eisinger, Proc. Natl. Acad. Sci. U.S.A. 59, 46 (1968).

${ }^{3}$ R. O. Rahn, Nucleic Acids Res. 3, 879 (1976).

${ }^{4}$ V. I. Danilov, O. N. Slyusarchuk, J. L. Alderfer, J. J. P. Stewart, and P. R. Callis, Photochem. Photobiol. 59, 125 (1994).

${ }^{5}$ T. Schultz, E. Samoylova, W. Radloff, I. V. Hertel, A. L. Sobolewski, and W. Domcke, Science 306, 1765 (2004).

${ }^{6}$ E. Samoylova, V. R. Smith, H. H. Ritze, W. Radloff, M. Kabelac, and T. Schultz, J. Am. Chem. Soc. 128, 15652 (2006).

${ }^{7}$ A. L. Sobolewski and W. Domcke, Chem. Phys. 294, 73 (2003).

${ }^{8}$ J. R. Roscioli and D. W. Pratt, Proc. Natl. Acad. Sci. U.S.A. 100, 13752 (2003).

${ }^{9}$ R. Wu and B. Brutschy, J. Phys. Chem. A 108, 9715 (2004).

${ }^{10}$ S. Perun, A. L. Sobolewski, and W. Domcke, J. Phys. Chem. A 110, 9031 (2006)

${ }^{11}$ A. L. Sobolewski, W. Domcke, and C. Hättig, Proc. Natl. Acad. Sci. U.S.A. 102, 17903 (2005).

${ }^{12}$ T. Yanai, D. P. Tew, and N. C. Handy, Chem. Phys. Lett. 393, 51 (2004); Y. Tawada, T. Tsuneda, S. Yanagisawa, T. Yanai, and K. Hirao, J. Chem. Phys. 120, 8425 (2004).

${ }^{13}$ F. Santoro, V. Barone, and R. Improta, J. Am. Chem. Soc. 131, 15232 (2009).

${ }^{14}$ A. Tsolakidis and E. Kaxiras, J. Phys. Chem. A 109, 2373 (2005).

${ }^{15}$ D. Varsano, R. Di Felice, M. A. L. Marques, and A. Rubio, J. Phys. Chem. B 110, 7129 (2006).

${ }^{16}$ DALTON, release 2.0, a molecular electronic structure program (2005), http://www.kjemi.uio.no/software/dalton/dalton.html.

${ }^{17}$ M. J. Frisch, G. W. Trucks, H. B. Schlegel et al., GAUSSIAN 03, Revision B.02, Gaussian, Inc., Pittsburgh, PA, 2003.

${ }^{18}$ A. J. Marks and D. L. Thompson, J. Chem. Phys. 96, 1911 (1992).

${ }^{19}$ W. K. Hastings, Biometrika 57, 97 (1970).

${ }^{20}$ E. Samoylova, W. Radloff, H. H. Ritze, and T. Schultz, J. Phys. Chem. A 113, 8195 (2009).

${ }^{21}$ G. Villani, Chem. Phys. 316, 1 (2005).

${ }^{22}$ M. Marazzi, U. Sancho, O. Castaño, W. Domcke, and M. Frutos, J. Phys. Chem. Lett. 1, 425 (2010).

${ }^{23}$ M. Olivucci, Theoretical and Computational Photochemistry, Vol. 16 (Elsevier, Amsterdam, 2005). 